\title{
Nonexistence of Normal Quintic Abelian Surfaces in $P^{3}$
}

\author{
Iku NAKAMURA and Yumiko UMEZU \\ Hokkaido University and Toho University \\ (Communicated by S. Endo)
}

\section{Introduction.}

Normal surfaces $X_{d}$ of degree $d$ in the complex projective 3-space $\boldsymbol{P}^{3}$ have simple birational structure if $d$ is small: $X_{1}$ and $X_{2}$ are rational, and $X_{3}$ is birationally equivalent to a ruled surface (for further details, see [B-W], [H-W]), since in general $K_{X_{d}} \simeq$ $\mathcal{O}_{X_{d}}(d-4)$. Moreover $X_{4}$ is birationally equivalent to either a ruled or a $\mathrm{K} 3$ surface ([Um1]).

To the contrary, various $X_{d}$ may occur if $d \geq 5$. If the singularity of $X_{d}$ is mild, then $X_{d}$ is birationally equivalent to a surface of general type, while $X_{d}$ may be birationally equivalent to a ruled surface if it has severe singularity. Moreover there are examples of $X_{5}$ which are birationally equivalent to $\mathrm{K} 3$ surfaces, Enriques surfaces or general elliptic surfaces ([I], [Yan], [St], [K], [Um2], [Um3], [Um4]). This leads us to the question whether there exists an $X_{d}$ which is birationally an abelian or a hyperelliptic surface or not. The purpose of this note is to answer this question in the case of $d=5$. We prove:

MAIN THEOREM. No normal quintic surface in $\boldsymbol{P}^{3}$ is birationally equivalent to an abelian or a hyperelliptic surface.

Our proof of the theorem goes as follows. First we note that if a normal quintic surface $X=X_{5}$ is birationally an abelian or a hyperelliptic surface, then its minimal resolution $\tilde{X}$ is an at most 5-fold blowing-up $\mu: \tilde{X} \rightarrow \bar{X}$ of the non-singular minimal model $\bar{X}$. On the other hand, the pull-back of $K_{X}$ to $\tilde{X}$ minus $K_{\tilde{X}}$ is an effective divisor $\tilde{D}$, which reflects the property of the singularity of $X$ fairly well. Such property of $\tilde{D}$ and the condition of $\mu_{*} \tilde{D}$ as a divisor on an abelian or hyperelliptic surface finally lead us in every case to a contradiction.

CONJECTURE. No normal hypersurface in $\boldsymbol{P}^{3}$ is birationally equivalent to an abelian surface.

Also for hyperelliptic surfaces we raise: 
Problem. Are there normal hypersurfaces in $\boldsymbol{P}^{3}$ which are birationally hyperelliptic surfaces?

\section{§1. Preliminaries.}

In this section we summarize some results from local theory of surface singularity, which we will use later.

Let $(Y, y)$ be a numerically Gorenstein normal surface singularity, $\pi: \tilde{Y} \rightarrow Y$ its minimal resolution and $A$ the exceptional set: $A=\pi^{-1}(y)$. Then there is an effective divisor $D$ with $\operatorname{supp} D \subseteq A$ such that $\omega_{\tilde{Y}} \equiv \mathcal{O}_{\tilde{Y}}(-D)$. Let $p_{a}$ or $p_{g}$ stand for the arithmetic or the geometric genus of $(Y, y)$ respectively. By definition ([W]),

$$
p_{a}=\sup _{\substack{D^{\prime}>0 \\ \operatorname{supp}\left(D^{\prime}\right) \subseteq A}} p_{a}\left(D^{\prime}\right), \quad p_{g}=\operatorname{dim} R^{1} \pi_{*} \mathcal{O}_{\tilde{Y}}
$$

It is known (cf. [A]) that the following conditions are equivalent:

(i) $D \neq 0$ (ii) $\operatorname{supp} D=A$ (iii) $p_{a}>0$ (iv) $p_{g}>0$.

Lemma 1.1 (Y. Koyama). $p_{a} \leq-D^{2} / 8+1$. In particular, if $D^{2} \geq-7$, then $p_{a} \leq 1$.

Proof. See [Um5].

REMARK 1.2. If $D^{2}=-8$ and $p_{a}=2$, then $D / 2$ is an integral and the unique divisor on $A$ whose arithmetic genus is equal to 2 .

In what follows (except for Corollary 1.5) we assume moreover that $p_{a}=1$, i.e. our singularity $(Y, y)$ is elliptic $([\mathrm{W}])$. Then Yau (for the minimal good resolution) and Tomari (for any resolution) defined the elliptic sequence $\left\{Z_{1}, \cdots, Z_{l}\right\}$ as follows: Let $E$ denote the minimal elliptic cycle of Laufer [L], i.e., $E$ is the minimal effective divisor such that $\operatorname{supp} E \subseteq A$ and $p_{a}(E)=1$. For $Z_{1}$ we take the fundamental cycle. Suppose that we have defined $Z_{1}, \cdots, Z_{k}$. If $Z_{k} E<0$, we define $\left\{Z_{1}, \cdots, Z_{k}\right\}$ as the elliptic sequence: $l=k$. Assume $Z_{k} E=0$. Then let $B_{k+1}$ denote the connected component containing $E$ of the sum of the components $A_{i}$ of $A$ satisfying $Z_{k} A_{i}=0$. We define $Z_{k+1}$ to be the fundamental cycle of $B_{k+1}$. Since supp $Z_{k} \supsetneqq \operatorname{supp} Z_{k+1}$, the elliptic sequence $\left\{Z_{1}, \cdots, Z_{l}\right\}$ is defined as a finite sequence. The following results for the minimal resolution will play an important role later.

THEOREM 1.3 ([T], [Yau]). (i) $D=\sum_{i=1}^{l} Z_{i}$. (ii) $Z_{l}=E . \quad$ (iii) $p_{g} \leq l$.

From this theorem, we obtain the following

Corollary 1.4. $p_{g} \leq-D^{2}$.

COROLlaRY 1.5. Let $(Y, y)$ be a numerically Gorenstein normal surface singularity of geometric genus $p_{g}$, and $\pi: \tilde{Y} \rightarrow Y$ its minimal resolution. Assume that the exceptional set $\pi^{-1}(y)$ consists of a chain of curves $A_{0}=E, A_{1}, \cdots, A_{m}(m \geq 1)$ with $p_{a}(E)=1, p_{a}\left(A_{i}\right)=0$ 
$(1 \leq i \leq m)$. Then we have

(i) $E^{2}=-1$,

(ii) $m \geq p_{g}-1$ and $A_{i}^{2}=-2$ for $1 \leq i \leq p_{g}-2$.

Proof. The fundamental cycle $Z_{1}$ coincides with $\pi^{-1}(y)$ with reduced structure, hence $p_{a}\left(Z_{1}\right)=1$, and so $(Y, y)$ is an elliptic singularity ([W]). Then $E$ is the minimal elliptic cycle. Theorem 1.3 implies that for every $i, Z_{i}$ contains more than $p_{g}-i$ components. In particular, for $i=1$, we get $m \geq p_{g}-1$; for $i=2, Z_{1} E=0$ and $Z_{1} A_{i}=0$ $\left(1 \leq i \leq p_{g}-2\right)$, which proves $E^{2}=-1$ and $A_{i}^{2}=-2\left(1 \leq i \leq p_{g}-2\right)$.

\section{§2. Properties of divisors on the resolution.}

Let $X$ be a normal quintic surface in $P^{3}$. Let $\pi: \tilde{X} \rightarrow X$ denote the minimal resolution of $X, H$ a general hyperplane section of $X$ and $\tilde{H}$ its pull-back on $\tilde{X}$. Then there exists a unique effective divisor $\tilde{D}$ on $\tilde{X}$ such that $K_{\tilde{X}}=\tilde{H}-\tilde{D}$. This divisor $\tilde{D}$ is supported on the exceptional sets of $\pi$ which correspond to singularities with positive geometric genus. Let $\mu: \tilde{X}=X_{n} \stackrel{\mu_{n}}{\longrightarrow} X_{n-1} \stackrel{\mu_{n-1}}{\longrightarrow} \cdots \stackrel{\mu_{1}}{\longrightarrow} X_{0}=\bar{X}$ be the sequence of blowdowns obtaining a non-singular minimal model $\bar{X}$ of $\tilde{X}, \mu_{i}^{\prime}$ the induced morphism $\tilde{X} \rightarrow X_{i}$ $(0 \leq i \leq n)$, and $E_{i}(1 \leq i \leq n)$ the total transform on $\tilde{X}$ of the exceptional curve of the blow-up $\mu_{i}$. In what follows we fix our notations as above and assume moreover that $\bar{X}$ is either an abelian or a hyperelliptic surface.

LEMMA 2.1. $\widetilde{D}^{2}=-n-5$ and $1 \leq n \leq 5$. Moreover, if $n=5$ and if $\Gamma$ is a rational curve on $\tilde{X}$, then either

(i) $\tilde{H} \Gamma=1$ ( $\Gamma$ is not exceptional for $\pi), \tilde{D} \Gamma=2, \Gamma^{2}=-1$, or

(ii) $\tilde{H} \Gamma=0(\Gamma$ is exceptional for $\pi), \tilde{D} \Gamma=0, \Gamma^{2}=-2$.

Proof. Since $\bar{X}$ has a numerically trivial canonical bundle, $-n=K_{\tilde{X}}^{2}=(\tilde{H}-\tilde{D})^{2}=$ $5+\tilde{D}^{2}$, and hence $\tilde{D}^{2}=-n-5$. Since each $E_{i}$ contains at least one $(-1)$-curve and $\tilde{X}$ is the minimal resolution, we have $\tilde{H} E_{i}>0$, and so $5=\tilde{H}^{2}=\tilde{H}(\tilde{H}-\tilde{D})=\tilde{H} K_{\tilde{X}}=$ $\sum_{i=1}^{n} \tilde{H} E_{i} \geq n . n \geq 1$ since $\tilde{H}-\tilde{D} \not \equiv 0$. Note that any rational curve on $\tilde{X}$ is a component of $E_{i}$ for some $i$ since $\bar{X}$ contains no rational curve. Assume $n=5$. Then $\tilde{H} E_{i}=1(1 \leq i \leq 5)$. Hence, for each $i$, there exists a unique component $\Gamma_{i}$ in $E_{i}$, with multiplicity 1 , such that $\tilde{H} \Gamma_{i}=1$, and other components of $E_{i}$ are exceptional for $\pi$ and so have non-positive intersection number with $\tilde{D}$. Since $\Gamma_{i}$ is a $(-1)$-curve, $-1=K_{\tilde{X}} \Gamma_{i}=(\tilde{H}-\tilde{D}) \Gamma_{i}$, hence $\tilde{D} \Gamma_{i}=2$. By $-1=K_{\tilde{X}} E_{i}=K_{\tilde{X}} \Gamma_{i}+K_{\tilde{X}}\left(E_{i}-\Gamma_{i}\right)=-1+\tilde{D}\left(E_{i}-\Gamma_{i}\right)$, we see that any component $\Gamma$ in $E_{i}-\Gamma_{i}$ satisfies $\tilde{D} \Gamma=0$ and so $\Gamma^{2}=-2$.

LEMMA 2.2. For each $i(1 \leq i \leq n)$, the center of the blow-up $\mu_{i}$ lies on the singular locus of $\left(\mu_{i-1}^{\prime}\right)_{*} \tilde{D}$.

Proof. Since $-1=K_{\tilde{X}} E_{i}=\tilde{H} E_{i}-\tilde{D} E_{i}$ and $\tilde{H} E_{i}>0$, we have $\tilde{D} E_{i} \geq 2$, which implies the Lemma. 
LEMMA 2.3. $\operatorname{dim} R^{1} \pi_{*} \mathcal{O}_{\tilde{X}}=5$.

Proof. From the exact sequence accociated with the Leray spectral sequence:

$$
0 \rightarrow H^{1}\left(X, \mathcal{O}_{X}\right) \rightarrow H^{1}\left(\tilde{X}, \mathcal{O}_{\tilde{X}}\right) \rightarrow R^{1} \pi_{*} \mathcal{O}_{\tilde{X}} \rightarrow H^{2}\left(X, \mathcal{O}_{X}\right) \rightarrow H^{2}\left(\tilde{X}, \mathcal{O}_{\tilde{X}}\right) \rightarrow 0,
$$

we have $\operatorname{dim} R^{1} \pi_{*} \mathcal{O}_{\tilde{X}}=4+q(\tilde{X})-p_{g}(\tilde{X})=5$.

COROllary 2.4. Let $\tilde{D}=\tilde{D}_{1}+\cdots+\tilde{D}_{s}$ be the decomposition of $\tilde{D}$ into its connected components. Then

$$
\sum_{i=1}^{s} h^{0}\left(\mathcal{O}_{\tilde{D}_{i}}\right)=5
$$

Proof. Let $y_{i}$ denote the singular point on $X$, which is obtained by contracting $\tilde{D}_{i}$. Let $Y_{i} \subset X$ be a Stein neighbourhood of $y_{i}, \tilde{Y}_{i}=\pi^{-1}\left(Y_{i}\right)$ and $\pi_{i}=\pi_{\mid} \tilde{Y}_{i}$. Note that $\omega_{\tilde{Y}_{i}} \simeq \mathcal{O}_{\tilde{Y}_{i}}\left(-\tilde{D}_{i}\right)$. Then we have $h^{0}\left(\mathcal{O}_{\tilde{D}_{i}}\right)=\operatorname{dim} R^{1}\left(\pi_{i}\right)_{*} \mathcal{O}_{\tilde{Y}_{i}}$, so that the Corollary follows from Lemma 2.3. In fact, consider the natural exact sequence:

$$
H^{1}\left(\mathcal{O}_{\tilde{Y}_{i}}\left(-\tilde{D}_{i}\right)\right) \rightarrow H^{1}\left(\mathcal{O}_{\tilde{Y}_{i}}\right) \rightarrow H^{1}\left(\mathcal{O}_{\tilde{D}_{i}}\right) \rightarrow 0 .
$$

We see that the first term vanishes by Grauert-Riemenschneider's theorem. The second term is isomorphic to $R^{1}\left(\pi_{i}\right)_{*} \mathcal{O}_{\tilde{Y}_{i}}$, and the third is dual to $H^{0}\left(\mathcal{O}_{D_{i}}\right)$.

LEMMA 2.5. Let $D$ be a connected divisor on $\tilde{X}$ with negative intersection matrix. If all irreducible components of $D$ are rational curves, then the contraction of $D$ is at worst rational singularity.

Proof. Since $\bar{X}$ contains no rational curve, the support of $D$ is contained in a divisor which is contracted to a non-singular point (by $\mu$ ). Hence the geometric genus of the contraction of $D$ vanishes.

LEMMA 2.6. Let $C$ and $C^{\prime}$ be irreducible curves on an abelian surface [resp. a hyperelliptic surface] $S$. Then

(i) $C^{2}$ is an even non-negative integer,

(ii) $C^{2}=0$ if and only if $C$ is a non-singular elliptic curve,

(iii) if the desingularization of $C$ is an elliptic curve, then $C$ itself is smooth,

(iv) if $C C^{\prime}=0$, then $C^{2}=C^{\prime 2}=0$ and $C$ and $C^{\prime}$ are algebraically equivalent [resp. if $C C^{\prime}=0$, then $C^{2}=C^{\prime 2}=0$ and $C \equiv q C^{\prime}$ for some positive $\left.q \in Q\right]$,

(v) if $C$ and $C^{\prime}$ are elliptic curves, then they intersect transversally.

ProOf. Since $S$ has trivial or numerically trivial canonical sheaf, $p_{a}(C)=C^{2} / 2+1$. Moreover $S$ contains no rational curves, whence (i) and (ii). For (iii)-(v), we first assume that $S$ is an abelian surface. Then (iii) is a special case of [Ue, Theorem 10.3]. (iv) If $C C^{\prime}=0$, then neither $C$ nor $C^{\prime}$ is ample, and so $C^{2}=C^{\prime 2}=0$. Moreover $C^{\prime}$ is a fiber of the quotient morphism $S \rightarrow S / C$. Hence $C$ and $C^{\prime}$ are algebraically equivalent. Finally, if two elliptic curves $C$ and $C^{\prime}$ intersect, then the morphism $C^{\prime} \rightarrow S / C$ is finite and 
unramified, and hence (v). Suppose next that $S$ is a hyperelliptic surface. Then there is a finite unramified covering $f: \tilde{S} \rightarrow S$ where $\tilde{S}$ is an abelian surface. Notice that any unramified cover of an elliptic curve is a disjoint union of elliptic curves. Hence (v) is clear. (iii) Let $\widetilde{C} \rightarrow C$ denote the desingularization of $C$. Then $\tilde{S} \times{ }_{S} \tilde{C}$ is the resolution of $f^{-1}(C)$. Since $\tilde{S} \times{ }_{S} \tilde{C}$ is a disjoint union of non-singular elliptic curves, we see that $f^{-1}(C)$ itself is non-singular, and hence so is $C$. (iv) The former part is proved in the same way as in the abelian case. Moreover, $C^{2}=C^{2}=0$ and $C C^{\prime}=0$ mean that $[C]$ and $\left[C^{\prime}\right]$ are not linearly independent in $N S(S) \otimes Q$.

\section{§3. Reduction to the case with only elliptic singularities.}

We use the notations in $\S 2$, and assume that $\bar{X}$ is either an abelian or a hyperelliptic surface. In this section we will prove that there exists on $X$ no singularity with arithmetic genus greater than 1 . We first notice by Lemma 2.5 that every connected component of $\tilde{D}$ contains a non-rational curve.

(3.1) Assume that there exists in $\tilde{D}$ an irreducible curve $D_{1}$ with $p_{a}\left(D_{1}\right) \geq 2$. Let $D$ denote the connected component of $\tilde{D}$ containing $D_{1}$ and $D^{\prime}$ the sum of the other components: $\tilde{D}=D+D^{\prime}$. By Lemma 1.1 and 2.1 , we have $-10 \leq D^{2} \leq-8$ and the arithmetic genus of the singularity corresponding to $D$ is equal to 2 . Hence, by Lemma 2.6 (iii), $D_{1}$ is a non-singular curve of genus 2 and the other components of $D$, if exist, are all non-singular rational curves. Since $0 \geq D^{\prime 2} \geq-2$, all singular points corresponding to $D^{\prime}$ are elliptic singularities.

Case 3.1.1. $D^{2}=-8$ : Remark 1.2 says that $D=2 D_{1}$ and $D_{1}^{2}=-2$. The exact sequence

$$
0 \rightarrow \mathcal{O}_{D_{1}}\left(-D_{1}\right) \rightarrow \mathcal{O}_{2 D_{1}} \rightarrow \mathcal{O}_{D_{1}} \rightarrow 0
$$

shows

$$
h^{0}\left(\mathcal{O}_{D}\right)=h^{0}\left(\mathcal{O}_{D_{1}}\left(-D_{1}\right)\right)+h^{0}\left(\mathcal{O}_{D_{1}}\right)=h^{0}\left(\omega_{D_{1}}\right)+1=3 .
$$

Hence, by Corollary 2.4 and its proof, it follows that $D^{\prime}$ corresponds to either one singular point with geometric genus equal to 2 or two singular points both of which have geometric genus 1. Hence $D^{\prime 2}=-2, n=5$ (Corollary 1.4 and Lemma 2.1). By Lemma 2.6, we have $\left(\mu_{*} D\right)^{2}>0$ and $\left(\mu_{*} D\right)\left(\mu_{*} D^{\prime}\right)>0$ and so there is a chain of rational curves $\Gamma_{1}, \cdots, \Gamma_{k}$ on $\tilde{X}$ such that $\Gamma_{i} \not \tilde{D}(1 \leq i \leq k)$ and $\Gamma_{1} D_{1}>0, \Gamma_{k} D^{\prime}>0$. Both $\Gamma_{1}$ and $\Gamma_{k}$ are not exceptional for $\pi$, and hence are (-1)-curves by Lemma 2.1. For $2 \leq i \leq k-1$, $\Gamma_{i}$ is either a $(-1)$-curve or else an exceptional curve for $\pi$, i.e., a (-2)-curve. Therefore it turns out that $k=1$ : there exists a $(-1)$-curve $\Gamma$ such that $\tilde{D} \Gamma=\left(2 D_{1}+D^{\prime}\right) \Gamma \geq 3$, which contradicts Lemma 2.1.

Case 3.1.2. $\quad D^{2}=-9$ : Let $D=m D_{1}+Y\left(Y ¥ D_{1}\right)$. Since $2=p_{a}\left(D_{1}\right)=\left(D_{1}^{2}-D_{1} D\right) / 2$ +1 , 


$$
D_{1} D=D_{1}^{2}-2 \leq-3
$$

Therefore

$$
-9=D^{2}=m D_{1} D+Y D \leq m D_{1} D=m\left(D_{1}^{2}-2\right) \leq-3 m,
$$

and so

$$
1 \leq m \leq 3
$$

If $m=1$, then $D_{1} D=D_{1}^{2}+D_{1} Y \geq D_{1}^{2}$, which contradicts (1).

If $m=2$, then, by (1), $-3 \geq D_{1} D=2 D_{1}^{2}+D_{1} Y$ and so $D_{1}^{2} \leq-2$. Hence $D_{1}^{2}=-2$ by (2). Hence, using (1), we have

$$
D_{1} Y=D_{1} D-2 D_{1}^{2}=D_{1}^{2}-2-2 D_{1}^{2}=0 \text {. }
$$

This implies $Y=0$ and so $D^{2}=\left(2 D_{1}\right)^{2}=-8$, a contradiction.

Assume $m=3$. Then $D_{1}^{2}=-1$ by (2) and hence $D_{1} Y=D_{1} D-3 D_{1}^{2}=0$ by (1), i.e. $Y=0: D=3 D_{1}$. Therefore

$$
h^{0}\left(\mathcal{O}_{D}\right) \leq h^{0}\left(\mathcal{O}_{D_{1}}\left(-2 D_{1}\right)\right)+h^{0}\left(\mathcal{O}_{2 D_{1}}\right)=h^{0}\left(\omega_{D_{1}}\right)+h^{0}\left(\mathcal{O}_{D_{1}}\left(-D_{1}\right)\right)+h^{0}\left(\mathcal{O}_{D_{1}}\right) \leq 4 .
$$

Hence we have $D^{\prime} \neq 0, n=5$, and are led to a contradiction as in Case 3.1.1.

Case 3.1.3. $D^{2}=-10$ : In this case $D=\tilde{D}$ and $n=5$. We set $\tilde{D}=m D_{1}+Y$ as before, where $\tilde{D} Y=0$ and $Y$ consists of $(-2)$-curves by Lemma 2.1 . Note that (1) in the previous case holds as well. Hence we have

$$
-10=\tilde{D}^{2}=m D_{1} \tilde{D}=m\left(D_{1}^{2}-2\right) \leq-3 m,
$$

therefore $m=1$ or 2 .

If $m=1$, then $D_{1} \tilde{D}=-10$ and $D_{1}^{2}=-8$, which is impossible because $D_{1} \tilde{D}=$ $D_{1}^{2}+D_{1} Y \geq D_{1}^{2}$.

If $m=2$, then $D_{1} \tilde{D}=-5$ and $D_{1}^{2}=-3$, hence $\tilde{D}=2 D_{1}+Y$ and $D_{1} Y=1$. This implies that $Y$ is a reduced irreducible $(-2)$-curve. But then we calculate

$$
h^{0}\left(\mathcal{O}_{\tilde{D}}\right)=h^{0}\left(\mathcal{O}_{D_{1}}\left(-D_{1}-Y\right)\right)+h^{0}\left(\mathcal{O}_{D_{1}+Y}\right)=h^{0}\left(\omega_{D_{1}}\right)+1=3,
$$

and so get a contradiction with Corollary 2.4 .

Hence we have proved with Lemma 2.6 that every non-rational component of $\tilde{D}$ is a non-singular elliptic curve.

(3.2) Suppose that $\tilde{D}$ has a connected component $D$ which contains two distinct non-singular elliptic curves $D_{1}$ and $D_{2}$. Then $D$ corresponds to a singularity with $p_{a}=2$ (Lemma 1.1). We set $\tilde{D}=D+D^{\prime}$.

Case 3.2.1. $D^{2}=-8$ : We can show a contradiction in a similar way as in Case 3.1.1, by taking a chain of reduced curves in $D$ connecting $D_{1}$ and $D_{2}$ instead of $D_{1}$. 
Case 3.2.2. $\quad D^{2}=-9$ : Set $D=m_{1} D_{1}+m_{2} D_{2}+Y, Y \nsupseteq D_{1}, D_{2}$. From

$$
D_{i}^{2}=D_{i} D=m_{i} D_{i}^{2}+m_{j} D_{1} D_{2}+D_{i} Y
$$

we have

$$
\left(1-m_{i}\right) D_{i}^{2}=m_{j} D_{1} D_{2}+D_{i} Y
$$

where $\{i, j\}=\{1,2\}$. Since $D$ is connected, the right hand side of (3) is positive, and so $m_{i} \geq 2(i=1,2)$. Also we have

$$
m_{1} D_{1}^{2}+m_{2} D_{2}^{2}=\left(m_{1} D_{1}+m_{2} D_{2}\right) D=D^{2}-D Y \geq D^{2}=-9 \text {. }
$$

Consider first the case of $D_{1} D_{2}>0$. Then $D_{1} D_{2}=1$ since $p_{a}=2$.

We first show that $D_{i}^{2} \leq-2(i=1,2)$. Assume to the contrary, say $D_{1}^{2}=-1$. Then (3) is reduced to

hence

$$
\begin{aligned}
& m_{1}-1=m_{2}+D_{1} Y, \\
& \left(1-m_{2}\right) D_{2}^{2}=m_{1}+D_{2} Y
\end{aligned}
$$

$$
\left(1-m_{2}\right) D_{2}^{2}=m_{2}+1+\left(D_{1}+D_{2}\right) Y .
$$

From (5), we get $m_{1} \geq m_{2}+1$. (6) implies $D_{2}^{2} \leq-2$, but if $D_{2}^{2}=-2$, then $m_{2} \geq 3$, and so $m_{1} \geq 4$, which contradicts (4). Therefore the unique possibility is $D_{2}^{2}=-3, m_{1}=3, m_{2}=2$ and $D_{1} Y=D_{2} Y=0$, i.e. $D=3 D_{1}+2 D_{2}$ with $D_{1}^{2}=-1, D_{2}^{2}=-3$. But then we have

$$
\begin{aligned}
h^{0}\left(\mathcal{O}_{D}\right) \leq & h^{0}\left(\mathcal{O}_{D_{1}}\left(-2 D_{1}-2 D_{2}\right)\right)+h^{0}\left(\mathcal{O}_{D_{2}}\left(-2 D_{1}-D_{2}\right)\right)+h^{0}\left(\mathcal{O}_{D_{1}}\left(-D_{1}-D_{2}\right)\right) \\
& +h^{0}\left(\mathcal{O}_{D_{1}+D_{2}}\right) \leq 4 .
\end{aligned}
$$

Hence it follows $D^{\prime} \neq 0$ and $n=5$, and then a contradiction as in Case 3.1.1. Thus we obtain $D_{1}^{2}, D_{2}^{2} \leq-2$.

By (4) we have $D_{1}^{2}=D_{2}^{2}=-2, m_{1}=m_{2}=2$ and $D Y=-1$. But then (3) implies $D_{1} Y=D_{2} Y=0$ and hence $Y=0$, a contradiction.

Therefore we obtain $D_{1} D_{2}=0$, in particular $D_{1} Y>0, D_{2} Y>0$. Then, by (3),

$$
\left(1-m_{i}\right) D_{i}^{2}=D_{i} Y \quad(i=1,2) .
$$

If $D_{1} Y=1$, then $m_{1}=2, D_{1}^{2}=-1$, and there exists a unique component $Y_{1}$ of $Y$ such that $Y_{1} D_{1}=1$ and that the multiplicity of $Y_{1}$ in $Y$ is 1 . Therefore

$$
\begin{aligned}
-2=Y_{1}^{2}-Y_{1} D & =Y_{1}\left(-2 D_{1}-m_{2} D_{2}-\left(Y-Y_{1}\right)\right) \\
& =-2-Y_{1}\left(m_{2} D_{2}+\left(Y-Y_{1}\right)\right)<-2 .
\end{aligned}
$$

Hence $D_{1} Y \geq 2, D_{2} Y \geq 2$.

Suppose $D_{i}^{2}=-1$. Then, since $\left(\mu_{*} D_{i}\right)^{2}=0$ (Lemma 2.6 (ii), (iii)), there exists a 
unique component $Y_{i}$ of $Y$ such that $D_{i} Y_{i}=1$ and every other component of $Y$ is disjoint from $D_{i}$. Moreover, we see from (7) that the multiplicity of $Y_{i}$ in $Y$ is $\mathrm{m}_{i}-1$, and that $m_{i} \geq 3$ since $D_{i} Y \geq 2$. We note that $\left(\mu_{*} D_{1}\right)\left(\mu_{*} D_{2}\right)>0$. Hence, if furthermore $D_{1}^{2}=D_{2}^{2}=-1$, we get $m_{1}=m_{2} \geq 3$ because in this situation $Y_{1}=Y_{2}$ in the notation above. Therefore, assuming $D_{1}^{2} \geq D_{2}^{2}$ in general, we have by (4) and (7) that there are the following possibilities:

$\begin{array}{lccccr} & D_{1}^{2} & D_{2}^{2} & m_{1} & m_{2} & D Y \\ \text { (i) } & -1 & -1 & 3 & 3 & -3 \\ \text { (ii) } & -1 & -1 & 4 & 4 & -1 \\ \text { (iii) } & -1 & -2 & 3 & 2 & -2 \\ \text { (iv) } & -1 & -2 & 3 & 3 & 0 \\ \text { (v) } & -1 & -3 & 3 & 2 & 0 \\ \text { (vi) } & -1 & -2 & 4 & 2 & -1 \\ \text { (vii) } & -1 & -2 & 5 & 2 & 0 \\ \text { (viii) } & -2 & -2 & 2 & 2 & -1\end{array}$

In (i) and (ii), there is a curve $Y_{1}$ of multiplicity $m_{1}-1 \geq 2$ in $Y$ with $D_{1} Y_{1}=$ $D_{2} Y_{1}=1$. Note that $Y_{1}^{2} \leq-3$, since if $Y_{1}^{2}=-2$ then $0=D Y_{1} \geq 2 m_{1}+\left(m_{1}-1\right)(-2)=2$. Hence (ii) is impossible, and in (i) we have $Y_{1}^{2}=-3$ by $D Y=-3$. But then $-1=D Y_{1} \geq$ $2 m_{1}+\left(m_{1}-1\right)(-3)=0$. In (iv) and (v), all components of $Y$ are $(-2)$-curves. There is a component $Y_{1}$ with multiplicity $m_{1}-1=2$ in $Y$ and $D_{1} Y_{1}=1$. Hence $\left(D-3 D_{1}-2 Y_{1}\right) Y_{1}=1$, and so there is a unique component $Y_{2}$ with multiplicity 1 in $Y$ such that $Y_{1} Y_{2}=1$. This implies $D=3 D_{1}+2 Y_{1}+Y_{2}$, which is absurd. In (vi)-(viii), where $D_{2}^{2}=-2$ and $m_{2}=2$, there is a curve $Y_{2}$ of multiplicity 2 in $Y$ with $D_{2} Y_{2}=1$. Since $D Y \geq-1, Y_{2}$ is a (-2)-curve. Hence there is a unique curve $Y_{3}$ in $D-2 D_{2}-2 Y_{2}$ of multiplicity 2 in it with $Y_{2} Y_{3}=1, Y_{3}$ is a (-2)-curve if $Y_{3} \leq Y$. Proceeding in this way, we find in (vi) and (vii) an infinite sequence $Y_{2}, Y_{3}, \cdots$ in $Y$; in (viii) $D=2\left(D_{2}+Y_{2}+\cdots+D_{1}\right)$, contradicting $D^{2}=-9$. Therefore it only remains the case (iii). Since then also $D_{2}^{2}=-2$ and $m_{2}=2$, we can start from $D_{2}$ in the same way as above and deduce $D=3 D_{1}+2 Y_{1}+\cdots+2 Y_{k}+2 D_{2}$, where $k \geq 1, Y_{1}^{2}=-3, Y_{2}^{2}=\cdots$ $=Y_{k}^{2}=-2$ and $D_{1}, Y_{1}, \cdots, Y_{k}, D_{2}$ form a chain. Hence we obtain

$$
\begin{aligned}
h^{0}\left(\mathcal{O}_{D}\right) \leq & h^{\mathrm{O}}\left(\mathcal{O}_{D_{1}}\left(-2 D_{1}-2 Y_{1}-\cdots-2 Y_{k}-2 D_{2}\right)\right) \\
& +h^{0}\left(\mathcal{O}_{D_{1}+Y_{1}+\cdots+Y_{k}+D_{2}}\left(-D_{1}-Y_{1}-\cdots-D_{2}\right)\right)+h^{0}\left(\mathcal{O}_{D_{1}+Y_{1}+\cdots+Y_{k}+D_{2}}\right) \\
\leq & 4,
\end{aligned}
$$

and so $D^{\prime} \neq 0, n=5$, hence a contradiction as in Case 3.1.1.

Case 3.2.3. $D^{2}=-10$ : Note first that $D=\tilde{D}$ and $n=5$. Set $\tilde{D}=m_{1} D_{1}+m_{2} D_{2}+Y$ as in Case 3.2.2. Then we have as before

$$
\begin{aligned}
& \left(1-m_{i}\right) D_{i}^{2}=m_{j} D_{1} D_{2}+D_{i} Y, \quad\{i, j\}=\{1,2\}, \\
& m_{1}, m_{2} \geq 2,
\end{aligned}
$$


and since $Y$ consists of $(-2)$-curves by Lemma 2.1,

$$
m_{1} D_{1}^{2}+m_{2} D_{2}^{2}=\left(m_{1} D_{1}+m_{2} D_{2}\right) \tilde{D}=\tilde{D}^{2}-\tilde{D} Y=\tilde{D}^{2}=-10 .
$$

Suppose $D_{1} D_{2}>0$. Then $D_{1} D_{2}=1$ since $p_{a}=2$, and so (8) is rewritten as

$$
\begin{aligned}
& \left(1-m_{i}\right) D_{i}^{2}=m_{j}+D_{i} Y, \quad\{i, j\}=\{1,2\}, \\
& m_{1}, m_{2} \geq 2 .
\end{aligned}
$$

If $D_{1}^{2}=-1$, then $m_{1}=m_{2}+1+D_{1} Y$ by (10). In particular $D_{2}^{2} \leq-2$. If furthermore $D_{2}^{2} \leq-3$, then $D_{2}^{2}=-3, m_{1}=4$ and $m_{2}=2$ by (9), which is not compatible with (10). Hence $D_{2}^{2}=-2$, and we see with (9) and (10) that only $\left(m_{1}, m_{2}\right)=(4,3)$ is possible. The case of $D_{i}^{2} \leq-2$ is easier. Then, assuming $D_{1}^{2} \geq D_{2}^{2}$, there are two possibilities:

$\begin{array}{lcccccc} & D_{1}^{2} & D_{2}^{2} & m_{1} & m_{2} & D_{1} Y & D_{2} Y \\ \text { (i) } & -1 & -2 & 4 & 3 & 0 & 0 \\ \text { (ii) } & -2 & -3 & 2 & 2 & 0 & 1\end{array}$

In (i) we get $Y=0: \tilde{D}=4 D_{1}+3 D_{2}$. But then $\tilde{D}$ can not be obtained from $\bar{X}$ by more than 3 blow-ups (Lemma 2.2 and Lemma 2.6 (ii), (iii)). In (ii) $Y$ is a reduced irreducible (-2)-curve since $D_{2} Y=1$ and $m_{2}=2$. This implies

$$
h^{0}\left(\mathcal{O}_{\tilde{D}}\right)=h^{0}\left(\mathcal{O}_{D_{1}+D_{2}}\left(-D_{1}-D_{2}-Y\right)\right)+h^{0}\left(\mathcal{O}_{D_{1}+D_{2}+Y}\right)=h^{0}\left(\omega_{D_{1}+D_{2}}\right)+1=3,
$$

which is impossible by Corollary 2.4 .

This proves $D_{1} D_{2}=0$ and so by (8)

$$
\left(1-m_{i}\right) D_{i}^{2}=D_{i} Y \quad(i=1,2) .
$$

Then we have, as in Case 3.2.2, $m_{i} \geq 2 ; m_{i} \geq 3$ if $D_{i}^{2}=-1 ; m_{1}=m_{2}$ if $D_{1}^{2}=D_{2}^{2}=-1$. We may assume that $D_{1}^{2} \geq D_{2}^{2}$ and that $m_{1} \leq m_{2}$ if $D_{1}^{2}=D_{2}^{2}$. Then, by (9), the possibilities are as follows:

$\begin{array}{lcccc} & D_{1}^{2} & D_{2}^{2} & m_{1} & m_{2} \\ \text { (i) } & -1 & -1 & 5 & 5 \\ \text { (ii) } & -1 & -2 & 4 & 3 \\ \text { (iii) } & -1 & -2 & 6 & 2 \\ \text { (iv) } & -1 & -3 & 4 & 2 \\ \text { (v) } & -2 & -2 & 2 & 3 \\ \text { (vi) } & -2 & -3 & 2 & 2\end{array}$

For (i), (ii) and (iv), let $Y_{0}=D_{1}, Y_{1}, \cdots, Y_{k}, Y_{k+1}=D_{2}$ denote the chain of curves in $\tilde{D}$ connecting $D_{1}$ and $D_{2}(k \geq 1)$, and $l_{j}$ the multiplicity of $Y_{j}$ in $\tilde{D}$. Since $D_{1}^{2}=-1$, $l_{1}=m_{1}-1$. Moreover, since $Y_{j}$ is a $(-2)$-curve for $1 \leq j \leq k$, we have $l_{j}-1 \geq l_{j+1}$ for $0 \leq j \leq k$, and so $m_{1}-k-1 \geq m_{2}$. Hence (i) and (ii) are impossible. In (iv) we have $k=1$, hence $\tilde{D}=4 D_{1}+3 Y_{1}+2 D_{2}$. But then there exists a $(-1)$-curve $\Gamma$ on $\tilde{X}$ such that $\Gamma Y_{1}>0$ and so $\tilde{D} \Gamma=3$, which contradicts Lemma 2.1. Replace $D_{1}$ and $D_{2}$ in (iii). Then for 
each of the remaining cases we notice that $D_{1}^{2}=-2$ and $m_{1}=2$. Let $Y_{0}, Y_{1}, \cdots, Y_{k}, Y_{k+1}$ be as above. Then we have $\tilde{D}=2 D_{1}+2 Y_{1}+\cdots+2 Y_{k}+2 D_{2}+Y^{\prime}$, where $Y^{\prime}$ consists of (-2)-curves and is disjoint from $D_{1}, Y_{1}, \cdots, Y_{k}$. Hence only the case (vi) can occur. Since $D_{2} \tilde{D}=-3, D_{2} Y^{\prime}=1$, i.e., $Y^{\prime}$ is reduced and irreducible. But then there exists a (-1)-curve $\Gamma$ on $\tilde{X}$ such that $Y^{\prime} \Gamma=1$, hence $\tilde{D} \Gamma$ is odd, contradicting Lemma 2.1.

(3.3). By what we have proved, every connected component $D$ of $\tilde{D}$ is the union of an elliptic curve and some exceptional rational curves for $\mu$. Let $F$ be a non-trivial effective divisor on $\tilde{X}$ with $\operatorname{supp} F \cong \operatorname{supp} D$. Then $F=\mu^{*} \bar{F}+\sum_{i=1}^{n} a_{i} E_{i}$ where $\bar{F}=\mu_{*} F$ and $a_{i} \in Z(1 \leq i \leq n)$. Therefore

$$
\begin{aligned}
p_{a}(F) & =\frac{1}{2}\left(\mu^{*} \bar{F}+\sum_{i=1}^{n} a_{i} E_{i}\right)\left(\mu^{*} \bar{F}+\sum_{i=1}^{n} a_{i} E_{i}+\sum_{i=1}^{n} E_{i}\right)+1 \\
& =\frac{1}{2} \bar{F}^{2}+1-\frac{1}{2} \sum_{i=1}^{n} a_{i}\left(a_{i}+1\right) .
\end{aligned}
$$

Here $\bar{F}$ is a multiple of an elliptic curve and so $\bar{F}^{2}=0$ by Lemma 2.6. Moreover $a(a+1) \geq 0$ for any integer $a$. Hence $p_{a}(F) \leq 1$, and so the singularity corresponding to $D$ is elliptic.

\section{§4. The case with elliptic singularities.}

We continue to use the notations in $\S 2$ and assume that $\bar{X}$ is either an abelian or a hyperelliptic surface. To complete our proof of the Theorem, we will deduce a contradiction under the assumption that every connected component $D$ of $\tilde{D}$ corresponds to an elliptic singularity. Recall that then $D$ consists of a non-singular elliptic curve and possibly some rational curves.

(4.1) Assume that there exists a connected component $D$ of $\tilde{D}$ which contains a rational curve. This is equivalent to that $X$ has a singularity with $p_{g} \geq 2$. Set $\tilde{D}=D+D^{\prime}$ and let $D_{1}$ denote the unique elliptic curve in $D$. Then $D_{1}$ is the minimal elliptic cycle of $D$. In general, we note that $\mu_{*} \tilde{D}$ is numerically equivalent to $\mu_{*} \tilde{H}$, and hence is connected and ample. In particular $D^{\prime} \neq 0$.

Suppose first that the rational components of $D$ are not connected. Then $D_{1}^{2} \leq-2$ and there are exactly two connected components of rational curves because the number of blow-ups $n$ is bounded by 5 (Lemma 2.1). If one of them has length $\geq 2$, then the equality holds, the other has length 1 , and every rational curve in $D$ is a $(-2)$-curve. It follows that $D$ can not be contracted to a numerically Gorenstein singularity (Theorem 1.3 (ii)). Hence we have that $D=2 D_{1}+Y_{1}+Y_{2}$, where $Y_{i}$ is a rational curve with $D_{1} Y_{1}=D_{1} Y_{2}=1, Y_{1} Y_{2}=0$ and $D_{1}^{2}=-2$, and that the corresponding singularity has a geometric genus equal to 2 (Theorem 1.3 (i), (iii)). Hence the sum of the geometric genera of singularities corresponding to $D^{\prime}$ is equal to 3 (Lemma 2.3). Moreover, if 
$Y_{1}^{2} \leq-3$, then $n=5$, which contradicts Lemma 2.1. Hence we have $Y_{1}^{2}=Y_{2}^{2}=-2$, and so there exist rational curves $\Gamma_{1}$ and $\Gamma_{2}$ such that $Y_{i} \Gamma_{j}=\delta_{i j}$. If $n=4$, then $\Gamma_{1}$ and $\Gamma_{2}$ are $(-1)$-curves. There is on $\tilde{X}$ no rational curve other than $Y_{1}, Y_{2}, \Gamma_{1}$ and $\Gamma_{2}$, and so $D^{\prime}$ consists of three disjoint elliptic curves: $D^{\prime}=D_{2}+D_{3}+D_{4}$, each of which meets either $\Gamma_{1}$ or $\Gamma_{2}$. We may assume that $D_{2} \Gamma_{1}>0$ and $D_{3} \Gamma_{1}>0$. But then $\mu_{*} D_{2}$ intersects $\mu_{*} D_{3}$ tangentially, contradicting Lemma $2.6(\mathrm{v})$. If $n=5$, then $\Gamma_{1}$ and $\Gamma_{2}$ are also $(-1)$-curves by Lemma 2.1. There is another $(-1)$-curve $\Gamma_{3}$ which is disjoint from $D$, $\Gamma_{1}$ and $\Gamma_{2}$. Moreover $D^{\prime}=D_{2}+D_{3}+D_{4}$ as before. Since $\tilde{D} \Gamma_{j}=2$ and $D_{i} \Gamma_{j} \leq 1$ $(1 \leq i \leq 4,1 \leq j \leq 3)$ by Lemma 2.1 and 2.6 (iii), we can assume that the dual graph of $D_{1}, \cdots, D_{4}, Y_{1}, Y_{2}, \Gamma_{1}, \Gamma_{2}, \Gamma_{3}$ is as follows:

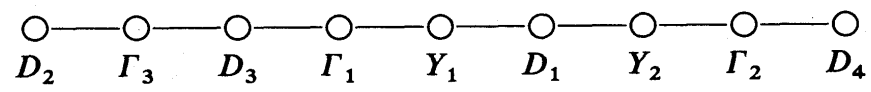

But then we obtain $\left(\mu_{*} D_{2}+\mu_{*} D_{3}\right)^{2}>0,\left(\mu_{*} D_{4}\right)^{2}=0$ and $\left(\mu_{*} D_{2}+\mu_{*} D_{3}\right)\left(\mu_{*} D_{4}\right)=0$, a contradiction to Hodge Index Theorem. It follows that there is a unique rational component $Y_{1}$ in $D$ which intersects $D_{1}$.

Suppose that there is a rational curve in $D$ which intersects more than two other components in $D$. From Lemma 2.1, we can deduce that the dual graph of $D$ is one of the following:

(i)

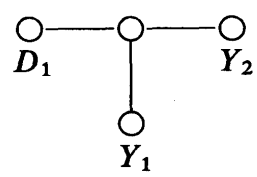

(ii)

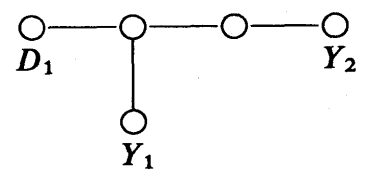

In (ii) we have $n=5$. Hence all rational components of $D$ are (-2)-curves (Lemma 2.1), and so there is a unique rational curve $\Gamma$, which is a (-1)-curve, except the components of $D . \Gamma$ intersects $Y_{1}$ or $Y_{2}$, and also every elliptic curve $D_{i}$ in $D^{\prime}$. Therefore $\mu_{*} D_{1}$ and $\mu_{*} D_{i}$ can not intersect transversally, which contradicts Lemma 2.6 (v). In (i), if $n=4$, then there is a $(-1)$-curve $\Gamma$, which plays a similar role as $\Gamma$ in (ii), and we are led to a contradiction. Assume $n=5$. Then every rational component in $\tilde{D}$ is a $(-2)$-curve, hence again there is a unique $(-1)$-curve $\Gamma_{1}$ which intersects $Y_{1}$ or $Y_{2}$. If $D^{\prime} \Gamma_{1}>0$, we get a contradiction as in (ii). If $D^{\prime} \Gamma_{1}=0$, then there is another $(-1)$-curve $\Gamma_{2}$ such that $D_{1} \Gamma_{2}>1, D^{\prime} \Gamma_{2}>0$. But the multiplicity of $D_{1}$ in $D$ is greater than 1 (Theorem 1.3), and so we have $D \Gamma_{2} \geq 2$, hence $\tilde{D} \Gamma_{2} \geq 3$, which contradicts Lemma 2.1. Therefore we conclude that $D$ consists of a chain $D_{1}, Y_{1}, \cdots, Y_{m}(m \geq 1)$ of an elliptic curve $D_{1}$ and rational curves $Y_{i}$.

Let $\tilde{D}=\tilde{D}_{1}+\cdots+\tilde{D}_{s}$ be the decomposition of $\tilde{D}$ into its connected components with $\tilde{D}_{1}=D$. Let $p_{i}$ denote the geometric genus of the singularity corresponding to $\tilde{D}_{i}$, $D_{i}$ the unique elliptic curve in $\tilde{D}_{i}$. We note that $D_{i}^{2}=-1$ if $p_{i} \geq 2$ (Corollary 1.5 (i)), and that $D_{i}^{2} \geq-3$ if $p_{i}=1$, since then $\tilde{D}_{i}=D_{i}$ is to be contracted to a simple elliptic hypersurface singularity ( $[\mathrm{Sa}])$. Set $C_{i}=\mu_{*} D_{i}$, then $C_{i}$ is also a non-singular elliptic curve. Recall that $C_{1}+\cdots+C_{s}$ is connected and ample. In particular $s \geq 2$ and there exists $i$ 
$(2 \leq i \leq s)$ such that $C_{1} C_{i}>0$. Since $D_{1}^{2}=-1$, we see that $C_{1} C_{i}=1$. We may assume that the point $P_{1}=C_{1} \cdot C_{i}$ is the center of the first blow-up $\mu_{1}$. By our assumption, the proper transform $Y_{1}$ on $\tilde{X}$ of $\mu_{1}^{-1}\left(P_{1}\right)$ is a component of $D$, and so $D_{i}^{2} \leq-2$, and hence $p_{i}=1$. Suppose that some $p_{j}(2 \leq j \leq s)$ is greater than 1 . Then we have $C_{1} C_{j}=0$, and so $C_{i} C_{j}>0$ for any $i$ such that $C_{1} C_{i}>0$, since $C_{1}+C_{i}$ is ample (or by Lemma 2.6 (iv)). Hence our assumption that $p_{j} \geq 2$ implies $D_{i}^{2} \leq-4$, which is impossible. Therefore we have $p_{i}=1$ for $2 \leq i \leq s$, and so $p_{1}+s-1=5$ (Lemma 2.3). We may assume that $P_{1} \in C_{i}\left(1 \leq i \leq s_{1}\right)$ and $P_{1} \notin C_{i}\left(s_{1}+1 \leq i \leq s\right)$ for some $s_{1}\left(2 \leq s_{1} \leq s\right)$. Since $C_{s_{1}+1}, \cdots, C_{s}$ are disjoint from $C_{1}$, they are also disjoint from each other. Hence $C_{2} \cap\left(\sum_{i=s_{1}+1}^{s} C_{i}\right)$ consists of greater than or equal to $s-s_{1}$ distinct points. This proves, with Corollary 1.5 (ii), $-3 \leq D_{2}^{2} \leq-p_{1}-\left(s-s_{1}\right)=s_{1}-6$. On the other hand, if $p_{1} \geq 3$, then $Y_{1}^{2}=-2$ by Corollary 1.5 (ii), and so $s_{1}=2$ (cf. Lemma 2.6 (v)), which is impossible. Hence we obtain $p_{1}=2, s=4$ and $3 \leq s_{1} \leq 4$. The last inequality implies $Y_{1}^{2} \leq-3$ and hence $n \leq 4$ (Lemma 2.1). If $s_{1}=3$, then we have by Theorem $1.3 \tilde{D}_{1}=2 D_{1}+Y_{1}$ with $Y_{1}^{2}=-3$, $D_{2}^{2}=D_{3}^{2}=-3$ and $D_{4}^{2}=-1$ :

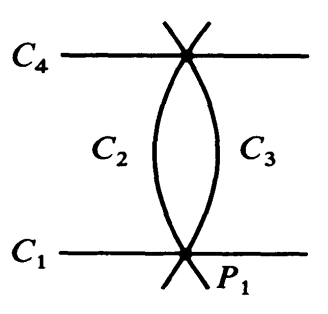

$\bar{X}$
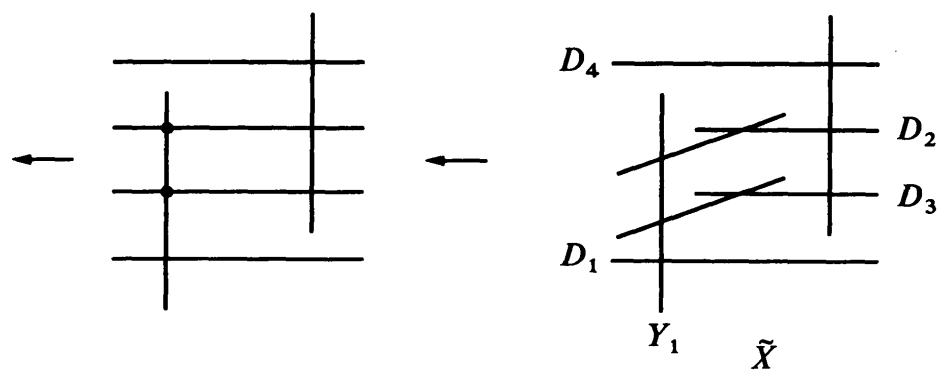

If $s_{1}=4$, then $\tilde{D}_{1}=2 D_{1}+Y_{1}$ with $Y_{1}^{2}=-4$ and $D_{2}^{2}=D_{3}^{2}=D_{4}^{2}=-2$ :

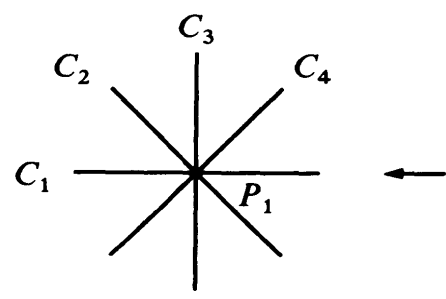

$\bar{X}$
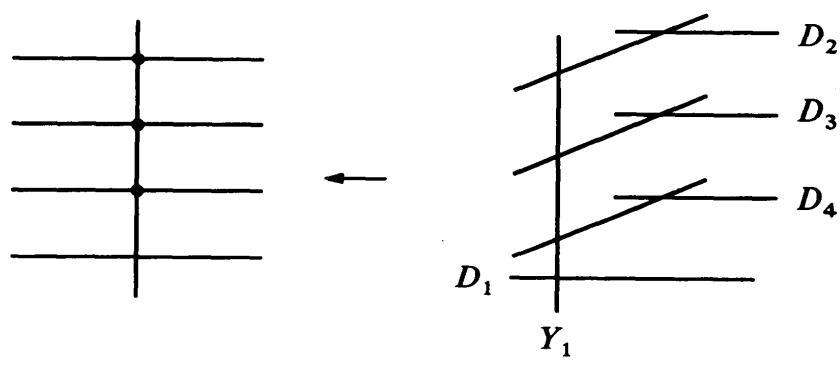

$\tilde{X}$

In both cases we obtain $\tilde{D}^{2}=-10$, contradictory to Lemma 2.1.

Thus we proved that $\tilde{D}$ has no rational components.

(4.2) Finally let us consider the case where $\tilde{D}$ consists of disjoint non-singular elliptic curves. Lemma 2.3 implies that $\tilde{D}$ has five components. Set $\tilde{D}=\sum_{i=1}^{5} D_{i}$ and $C=\mu_{*} D=\sum_{i=1}^{5} C_{i}$ where $C_{i}=\mu_{*} D_{i} . C_{i}$ and $D_{i}$ are non-singular elliptic curves $(1 \leq i \leq 5)$, $D_{i}$ 's are disjoint, but $C$ is connected. Let $P_{i}(1 \leq i \leq n)$ denote the center of the blow-up $\mu_{i}$. Then, by Lemma 2.2 and $2.6(\mathrm{v}), P_{1}, \cdots, P_{n}$ are not infinitely near each other, hence 
we may regard them as distinct points on $\bar{X}$. Set $k_{i}=$ mult $_{P_{i}} C$. Then $k_{i}$ is equal to the number of curves $C_{j}$ which pass through $P_{i}$. Lemma 2.2 says $k_{i} \geq 2(1 \leq i \leq n)$, and we may assume $5 \geq k_{1} \geq k_{2} \geq \cdots \geq k_{n} \geq 2$.

With these notations we have first from Lemma 2.1

$$
\sum_{i=1}^{n} k_{i}=n+5
$$

since $-n-5=\widetilde{D}^{2}=\sum_{j=1}^{5} D_{j}^{2}=\sum_{j=1}^{5} C_{j}^{2}-\sum_{i=1}^{n} k_{i}=-\sum_{i=1}^{n} k_{i}$. Next, let us show that $C_{i} C_{j}>0$ for any $i, j(i \neq j)$. Let $s$ denote the maximal number of components in $C$, which are disjoint each other. We may assume that $C_{1}, \cdots, C_{s}$ are disjoint. Lemma 2.6 (iv) implies $C_{j} \equiv q_{j} C_{1}$ for $2 \leq j \leq s$, where $q_{j}$ are some positive rational numbers. Hence we obtain by (11)

$$
\begin{aligned}
s(5-s) & \leq\left(\sum_{j=1}^{s} C_{j}\right)\left(\sum_{j=s+1}^{5} C_{j}\right) \\
& =\sum_{i ; P_{i} \in \bigcup_{j=1}^{s} C_{j}}\left(k_{i}-1\right) \leq \sum_{i=1}^{n}\left(k_{i}-1\right)=5,
\end{aligned}
$$

and so $s=1,4$ or 5. If $s=5$, then $C$ is not connected, which is excluded. If $s=4$, then $C_{5}$ meets $C_{1}, C_{2}, C_{3}$ and $C_{4}$, and hence $D_{5}^{2} \leq-4$, which is impossible since $D_{5}$ corresponds to a simple elliptic hypersurface singularity. Therefore $s=1$ as required.

Now we shall derive a contradiction for each $n(1 \leq n \leq 5$ by Lemma 2.1) from what we have proved.

$n=1$ : Clear since then $5 \geq k_{1}=n+5=6$.

$n=2$ : We have two possibilities: (i) $k_{1}=5, k_{2}=2$; (ii) $k_{1}=4, k_{2}=3$. In (i), all $C_{i}$ pass through $P_{1}$ and we may assume that $P_{2} \in C_{1}, C_{2}$ and $P_{2} \notin C_{3}, C_{4}, C_{5}$. Then the intersection form of $C_{1}, \cdots, C_{5}$ is as follows:

$$
\left(C_{i} C_{j}\right)=\left(\begin{array}{lllll}
0 & 2 & 1 & 1 & 1 \\
2 & 0 & 1 & 1 & 1 \\
1 & 1 & 0 & 1 & 1 \\
1 & 1 & 1 & 0 & 1 \\
1 & 1 & 1 & 1 & 0
\end{array}\right),
$$

which is clearly non-degenerate. But the Picard number of $\bar{X}$ is not greater than 4 since $\bar{X}$ is an abelian or a hyperelliptic surface, and so we get a contradiction. In (ii), we may assume that $P_{1} \notin C_{1}$, but then $C_{1}$ must meet every $C_{i}(2 \leq i \leq 5)$ away from $P_{1}$, that is at $P_{2}$, a contradiction.

$n=3$ : There are two possibilities: (i) $k_{1}=4, k_{2}=k_{3}=2$; (ii) $k_{1}=k_{2}=3, k_{3}=2$. In both cases we may assume $P_{1} \notin C_{1}$, and hence $C_{1}$ meets every $C_{i}(2 \leq i \leq 5)$ at $P_{2}$ or $P_{3}$, which is impossible for $k_{2}, k_{3}<5$ and $k_{2}+k_{3}<6$.

$n=4$ : We have $k_{1}=3, k_{2}=k_{3}=k_{4}=2$. Assuming $P_{1} \notin C_{1}$, we see that $C_{1}$ should meet every $C_{i}(2 \leq i \leq 5)$ at either $P_{2}, P_{3}$ or $P_{4}$, which is also impossible. 
$n=5: \quad$ In this last case, we have $k_{1}=\cdots=k_{5}=2$ and so the five curves $C_{1}, \cdots, C_{5}$ should meet each other at only five points $P_{1}, \cdots, P_{5}$ with multiplicity 2 , which is absurd.

Thus we have completed our proof of the Main Theorem.

\section{References}

[A] M. ARtin, On isolated rational singularities of surfaces, Amer. J. Math. 88 (1966), 129-136.

[B-W] J. W. BRUCE and C. T. C. WALL, On the classification of cubic surfaces, J. London Math. Soc. (2) 19 (1979), 245-256.

[H-W] F. Hidaka and $\mathrm{K}$. Watanabe, Normal Gorenstein surfaces with ample anti-canonical divisor, Tokyo J. Math. 4 (1981), 319-330.

[I] M. IsHIDA, On surfaces defined by a sum of four monomials, Proceedings of Symposium on Algebraic Geometry, Kinosaki 1982, 163-184 (in Japanese).

[K] Y. KIM, On normal quintic Enriques surfaces, Thesis, Univ. of Michigan (1991).

[L] H. LAUFER, On minimally elliptic singularities, Amer. J. Math. 99 (1977), 1257-1295.

[Sa] K. SAITo, Einfach-elliptische Singularitäten, Invent. Math. 23 (1974), 289-325.

[St] E. Stagnaro, Constructing Enriques surfaces from quintics in $\boldsymbol{P}_{K}^{3}$, Algebraic Geometry-Open Problems, Ravello 1982 (eds. C. Ciliberto et al.), Lecture Notes in Math. 997 (1983), Springer.

[T] M. Tomari, A $p_{g}$-formula and elliptic singularities, Publ. R. I. M. S. Kyoto Univ. 21 (1985), $297-354$.

[Ue] K. Ueno, Classification Theory of Algebraic Varieties and Compact Complex Spaces, Lecture Notes in Math. 439 (1975) Springer.

[Um1] Y. UMEZU, On normal projective surfaces with trivial dualizing sheaf, Tokyo J. Math. 4 (1981), 343-354.

[Um2] Y. UMEZU, Normal quintic surfaces which are birationally Enriques surfaces, preprint.

[Um3] Y. UmezU, On birational models of Enriques surfaces in $\boldsymbol{P}^{3}$, Proc. Japan Acad. 70 (1994), $137-139$.

[Um4] Y. UMEZU, Normal quintic surfaces with Kodaira dimension one (preprint).

[Um5] Y. UMEZU, Irregularity of quintic surfaces of general type, Tokyo J. Math. 17 (1994), $181-186$.

[W] P. WAGREICH, Elliptic singularities of surfaces, Amer. J. Math. 92 (1970), 419-454.

[Yan] J.-G. YANG, Characterizations of normal quintic K-3 surfaces, Trans. Amer. Math. Soc. 313 (1989), 737-751.

[Yau] S. S.-T. YAU, On maximally elliptic singularities, Trans. Amer. Math. Soc. 257 (1980), 269-329.

Present Addresses:

IKU NAKAMURA

Department of MATHEMatics, Hokkaido UNiversity,

SAPPORO, 060 JAPAN.

YUMIKO UMEZU

Department of Mathematics, School of Medicine, Toho University,

OMORI-NISHI, OTA-KU, TOKYO, 143 JAPAN. 